\title{
The Automated Acquisition of 'Topic Signatures for Text Summarization
}

\author{
Chin-Yew Lin and Eduard Hovy \\ Information Sciences Institute \\ University of Southern California \\ Marina dol lioy, CA 90292, USA \\ $\{c y l, h o v y\} @ i s i . e c h$
}

\begin{abstract}
In order to produce a good summary, one hats to iclentify the most relevant portions of a given text. Wo describe in this paper a method for antomatically training toplic signat.ures-sets of related words, with associated weights, organized around heal topics and illustrate with signatures we created with 6,194 TREC collection texts over 4 selacted topics. We describe the possible integration of topjo signatures with ontologies and its eviluaton on an automated text summarization system.
\end{abstract}

\section{Introduction}

'This paper describes the automated creation of what, we call topic signatures, constructs that can play a central rolo in automated text summarization and information retrieval. Topic signatures can be used to identify the presence of a complex concept--ia concept that consists of several related components in fixed relationships. Restanrant-visit, for example, involves at least the concepts menu, eat, pay, and possibly waiter, and Dragon Boat Festival (in Taiwan) involves the concents celemazs (a, talisman to ward of evil), moxa (something with the power of preventing pestilence and strongthening health), pictures of Chung Kuei (a nemesis of evil spirits), eggs standing on end, etc. Only when the concepts cooccur is one licensed to infer the complex concept; cat or moxa alone, for example, are not sufficient. At this time, we do not consider the interrelationships among the concepts.

Since many texts may describe all the components of a complex concept without ever explicitly mentioning the underlying complex concept--a a topic--itself, systems that have to identify topic(s), for: summarization or information retrieval, require a method of inferring conplex concepts from their componont words in the text.

\section{Related Work}

In late 1970's, Jo.Jong (DeJong, 1982) devoloped a system called FRLMP (Fast Reading Understanding and Memory Program) to skim newspaper storics and cxtract the main details. FRCMP uses a data structure called sketchy seript to organize its work knowledge. Each sketchy script is what FRLMP knows about what can occur in particular situations such as denonstrations, oarthquakes, labor strikes, and so on. FRUMP selects a particular slietchy script based on clues to styled events in news articles. In otluer words, FRUMP selects an empty template whose slots will be fillex on the fly as FRUMP reads a news article. A smmmary is generated based on what has been eaptured or fillod in the template.

The recent success of infomation extraction research has encomaged the FRUMP approach. The SUMMONS (SUMMirizing (Online NewS articles) system (Xrokeown and Radev, 1999) takes template outputs of information extraction systems developed for $\mathrm{MUC}$ conference and generating summaries of multiple news articles. FRUMP and SUMMONS both rely on prior knowlextge of their domains. However, to acepuire such prior knowledge is labor-intensive and time-cousuming. For cxample, the University of Massachusetts CIRCUS systene used in the MUC-3 (SAIC, 1998) terrorism domain reguired about bofo person-hours to define $\mathrm{x}$ traction parterns" (Riloff, 1996). In orker to make then practical, wo need to reduce the knowledge engincering bottencek and improve the portability of FRUMl' or SUMAONS-like systems.

Since the world contains thousands, or perhaps millions, of complex concepts, it is important to bo able to learn sketchy scripts or extraction patterns automatically from corpora no existing knowledge base contains nearly enough information. (Rilosf and forenzen, 1999) present a system AutoSlog-TS that. generates extraction pattems and learns lexical constraints intomatically from preclassified text to alleviate the knowledgo engineering bottleneck mentioned above. Although Riloff applied AntoSlog-TS

\footnotetext{
${ }^{1}$ We vewed shetely scripts and templates ats equivalent constructs in the sense that they specify high lovel entities and rolationships for specific topics

2 An extraction pattem is esontutially a case frame contains its trigger word, enabling conditions, variable slots, and slot constraints. CIRCUS uses a database of extraction patterns to parse texts (Rilof, 1996).
} 
to text catcgorization and information extraction, the concept of relevancy signatures introduced by her is very sirnilar to the topic signatures we proposed in this paper. Relevancy signatures and topic signatures are both trained on preclassified documents of specific topics and used to iclentify the presence of the larned topics in previously unseen documents. The main differences to our approach are: relevancy signatures require a parser. They are sentence-based and applied to text categorization. On the contrary, topic signatures only rely on corpus statistics, are document-based ${ }^{3}$ and used in text summarization.

In the next section, we describe the automated text summarization system SUMMARIST that we used in the experiments to provide the context of discussion. We then define topic signatures and detail the procedures for automatically constructing topic signatures. In Section 5, we give an overview of the corpus used in the evaluation. In Section 6 we present the experimental results and the possibility of enriching topic signatures using an existing ontology. Finally, we end this paper with a cons:lnsion.

\section{SUMMARIST}

SUMMARIST (Hovy and Lin, 1999) is a system designed to generate summaries of multilingilal input texts. At this time, SUMMARIST can process English, Arabic, Bahasa Indonesia, Japanese, Korean, and Spanish texts. It combines robust natural language processing methods (morphological transformation and part-of-speecl tagging), symbolic world knowledge, and information retricval techniques (term distribution and frequency) to achieve high robustness and better concept-level generalization.

The core of SUMMARIST is based on the following 'oquation':

summarization = topic identification +

topic interpretation + generation.

These three stages are:

Topic Identification: ldentify the most important (central) topics of the texts. SUMMARIST uses positional importance, topic signature, and term frequency. Importance based on discourse structure will be added later. 'This is the most developed stage in SUMMARIST.

Topic Interpretation: To fuse concepts such as waiter, menu, and food into ono generalized concept restaurant, we need more than the simple word aggregation used in traditional information retrieval. We have investigated concept

\footnotetext{
${ }^{3}$ We would like to use only the relevant parts of documents to generate topic signatures in the future. Text segmentation algorithms such as Text Tiling (Ilexrst, 1997) can be used to find subtopic segments in text.
}

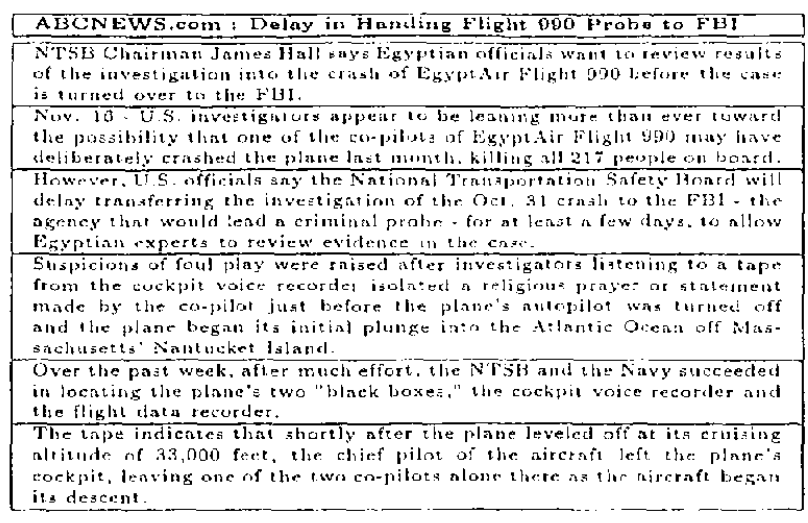

Iigure 1: A Nov. 161999 ABC News page summary generated by SUMMARIST.

combing and topic signatures to tackle the fusion problem.

Summary Generation: SUMMARIST can produce keyword and extract type summaries.

Figure 1 shows an ABC News page summary about EgyptAir Flight 990 by SUMMARIST. SUMMARIST employs several different heuristics in the topic identification stage to score terms and sentences. The score of a sentence is simply the sum of all the scores of content-bearing terms in the sentence. These heuristics are implemented in separate modules using inputs from preprocessing modules such as tokenizer, part-of-speech lagger, morphological analyzer, term frequency and tfidf weights cal. culator, sentence length calculator, and sentence location identifier. We only activate the position mod. ule, the tfidf module, and the topic signature module for comparison. We discuss the effectiveness of these modules in Section 6.

\section{Topic Signatures}

Before addressing the problem of world knowledge acquisition head-on, we decided to investigate what type of knowledge would be useful for summarization. After all, one can spend a lifetime acquiring knowledge in just a small domain. But what is the minimum amount of knowledge we need to enable effective topic identification as illustrated by the restaurant-visit example? Our iclea is simple. We would collect a set of terms that were typically highly correlated with a target concept from a preclassified corpus such as TREC collections, and then, during summarization, group the occurence of the related terms by the target concejt. For example, we would replace joint instances of table, menu, waiter, order, eat, pay, tip, and so on, by the single phrase restaurant-visit, in producing an indicative

\footnotetext{
"lerms can be stemmed words, bigrams, or trigrams.
} 
summary. Wo thus defined a topic signature as a family of related terms, as follows:

$$
\begin{aligned}
T S & =\{\text { topic, signature }\} \\
& =\left\{\text { topic },<\left(t_{1}, w_{1}\right), \ldots,\left(t_{n}, w_{n}\right)>\right\}
\end{aligned}
$$

where topic is the target concept and signature is a vector of related terms. Each $t_{t}$ is an term highly correlated to topic with association weight $w_{i}$. The number of related terms $n$. can be set ompirically according to a cutoff associated weight. We describe how to accuire related terms and their associated weights in the next section.

\subsection{Signature Term Extraction and Weight Estimation}

On the assumption that semantically related terms tend to co-occur, one can construct topic: signatures from preclassified text using the $\chi^{2}$ test, mutual information, or other standard statistic tests and information-theoretic measures. Instead of $\chi^{2}$, we use likelihood ratio (Dunning, 1993) $\lambda$, since $\lambda$ is more appropriate for sparse data than $\chi^{2}$ test and the quantity $-2 \log \lambda$ is asymptotically $\chi^{2}$ distributed ${ }^{5}$. Therefore, we can determine the confidence level for a specific $-2 \log \lambda$ value by looking up $\chi^{2}$ clistribution table and use the value to select an appropriate cutoff associated woight.

We have documents preclassified inte a set $R$ of relevant texts and a set $\tilde{R}$ of nomrelevant texts for a given topic. Assuming the following two hypotheses:

Hypothesis $1\left(H_{1}\right): P\left(\mathcal{R} \mid t_{i}\right)=p=P\left(\mathcal{R} \mid \bar{t}_{i}\right)$, i.e. the relevancy of a docinnent is independent of $t_{i}$

Hypothesis $2\left(H_{2}\right): P\left(\mathcal{R} \mid t_{i}\right)=p_{1} \neq p_{2}=$ $P\left(\mathcal{R} \mid \vec{t}_{i}\right)$, i.e. the presence of $t_{i}$ indicates strong relevancy assuming $p_{1} \gg p_{2}$.

and the following 2-by-2 contingency table:

\begin{tabular}{|c|c|c|}
\hline & $R$ & $\tilde{R}$ \\
\hline$t_{i}$ & $O_{11}$ & $O_{1 \cdot 2}$ \\
\hline$t_{i}$ & $O_{21}$ & $O_{2} \cdot 2$ \\
\hline
\end{tabular}

where $O_{11}$ is the frequency of term $t_{i}$ occurring in the relevant set, $O_{12}$ is the frequency of term $t_{i}$ or:curring in the nomrelevant set, $\mathrm{O}_{21}$ is the frecpuency of term $\tilde{t}_{i} \neq t_{i}$ occurring in the relevant set, $O_{2,2}$ is the frequency of term $\tilde{t}_{i} \neq t_{i}$ occurring in the nonrelevant set

Assming a binomial distribution:

$$
b(k ; n, x)==\left(\begin{array}{l}
n \\
k
\end{array}\right) x^{k}(1-x)^{(n-k)}
$$

5rhis assumes that the ratio is between the maximum likelihood estimate over a subpart of the parameter space and the maximum likelihood estimate over the entife parameter space. See (Manning and Schït\%e, 1999) pages 172 to 175 for details. then the likelihood for $M_{1}$ is:

$$
L\left(H_{1}\right)=b\left(O_{11} ; O_{11}+O_{12}, p\right) b\left(O_{21} ; O_{21}+O_{22}, p\right)
$$

and for $\mathrm{H}_{2}$ is:

$$
L\left(H_{2}\right)=b\left(O_{11} ; O_{11}+O_{12}, p_{1}\right) b\left(O_{21} ; O_{21}+O_{2 \cdot 2}, p_{2}\right)
$$

The $-2 \log \lambda$ value is then computed as follows:

$$
\begin{aligned}
& =-2 \log \frac{L\left(H_{1}\right)}{L\left(H_{2}\right)} \\
& -2 \log \frac{b\left(O_{11}: O_{11}+O_{12}, p\right) b\left(O_{21}: O_{21}+O_{22}, p\right)}{b\left(O_{11}: O_{11}+O_{12,1_{1}} b\left(O_{21}: O_{21}+O_{22}, p_{2}\right)\right.} \\
& -2\left(\left(O_{11}+O_{21}\right) \log p+\left(O_{12}+O_{22}\right) \log (1-p)-\right. \\
& \left(O_{11} \log p 1+O_{12} \log (1-n)+\theta_{21} \log m+\theta_{2} \log \left(1-\theta_{1}\right.\right. \\
& 2 N \times(H(\pi)-H(\pi \mid \tau)) \\
& 2 N \times I(\pi ; \tau)
\end{aligned}
$$

where $N=O_{11}+O_{12}+O_{21}+O_{22}$ is the total num. ber of term occurrence in the corpus, $\mathcal{H}(\mathcal{R})$ is the entropy of tems over relevant and nonrelevant sets of documents, $\mathcal{H}(\mathcal{R} \mid \mathcal{T})$ is the entropy of a given term over relevant and nomrelevant sets of documents, and $\mathcal{I}(\mathcal{R} ; \mathcal{T})$ is the mutual information between document relevancy and a given term. Equation 5 indicates that mutual information ${ }^{6}$ is an equivalent measure to likelihood ratio when we assume a binomial distribution and a $2-b y-2$ contingency table.

To create topic signature for a given topic, we:

1. classify documents as relevant or nonrelevant accorling to the given topic

2. compute the $-2 \log \lambda$ value using Equation 3 for cach term in the document collection

3. rank terms according to their $-2 \log \lambda$ value

4. select a confidence level from the $\chi^{2}$ distribution table; determine the crutoff associated weight and the number of terms to be included in the signaturesi

\section{The Corpus}

The training data derives from the Question and Answering summary evaluation data provided by TIPSTER-SUMMAC (Mani et al., 1998) that is a subset of the TREC collections. 'The TREC data is a collection of texts, classified into various topics, used for formal evaluations of information retrieval systems in a series of anmul comparisons. This data set contains essential text fragments (phrases, clanses, and sentences) which must be included in summaries to answer some 'TREC topics. These fragments are each judged by a human judge. As described in Section 3, SUMMARIST employs several independent modules to assign a score to each sentence, and then combines the scores to decide which sentences to extract from the input text. One can gauge the efficacy

\footnotetext{
6he mutual information is defined according to chapter 2 of (Cover and Thomat, 1991) and is not the pairwise mutual information used in (Chureh and Hanks, 1990).
} 


\begin{tabular}{|c|c|c|c|c|c|}
\hline \multicolumn{6}{|c|}{ Topic 10 Signature Terms of Topic 151 - Overcrawded Prisons } \\
\hline Thigtain & $-2 \operatorname{lng} \theta$ & 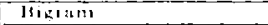 & $-2 \operatorname{ling} \lambda$ & 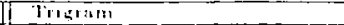 & $-2 \ln , \mathrm{A}$ \\
\hline jail & 10i 11.1 & Butaty jan! & $1+i+1,23$ & Bedetial contet onder & tis triil \\
\hline cintinty & (1) 621 & watly relwan & x.5.3+1 & 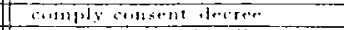 & 35.121 \\
\hline cowterionding & $14=3.44$ & state priation & $.13+2$ & 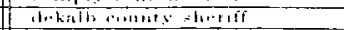 & 35131 \\
\hline (3) & $23+765$ & itate firisilum & is tititi & Hian in frituk & 35121 \\
\hline Fiteriff & $151.1+0$ & day fin... & $1.1 .16 \%$ & jom fiank halli & $3+121$ \\
\hline tate & 151.40 & gnil nowelenudins & 17i & prifoner county gatil & $3 \$ 121$ \\
\hline prifubet & $1.1 \% x$ & 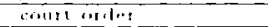 & riv. ining & Fast. proton couthy & 20.4 .4 \\
\hline prisem & $1.15 .30+i$ & $\mid$ Ineral jaxi! & $51+40$ & $t$ pee prison & $2+1.41$ \\
\hline ity & 133.47 & 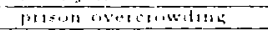 & 85375 & conculty jail tiate & $2+1311$ \\
\hline wetcruwdeal & 128.0105 & Ochetal rasility & $32 \sin$ & Theld locinl joil & 24341 \\
\hline \multicolumn{6}{|c|}{ Topic 10 Signature Terms of Topic 257 - Cigarete Consumption } \\
\hline Dimigratn & $2 \log \lambda \lambda$ & 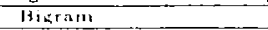 & $-2 \log 1$ & I] Ttigzatn & $-0 \log x$ \\
\hline 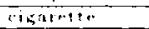 & 15titis & Tubaces industey & sio.itin & pholip monis rif & $28+6)$ \\
\hline Eotriction & $313.01 \%$ & fin casiatette & $8=420$ & 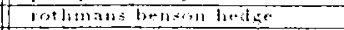 & $26 i !+i ! t$ \\
\hline stboking & 28.6196 & philipemorris & $5+107$ & lune cancer death & 2221.4 \\
\hline sincike & 1519.15 .4 & cisalette yobat & 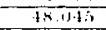 & Air firm die & $2 \frac{11 M}{31 M}$ \\
\hline Wothonans & $150.77 \mathrm{t}$ & n: internationat & $\sqrt{14.43}$ & 9 वा q & $21 .+16$ \\
\hline cesha & 1.18 .372 & wobaced moke & 4.269 & $\ln \sin \operatorname{ton}$ & 20220 \\
\hline $\operatorname{sentit}$ & 121.421 & जis patrick & 40.455 & 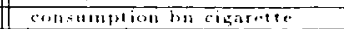 & 20.220 \\
\hline bati: & 113.8 .99 & cicateterecompany & 319.3100 & Great almerican simokeout & 20.2240 \\
\hline stinoker & 104.110 & cent markm. & 315.23 & lutes canter heart & 202261 \\
\hline biat & .99 .903 & 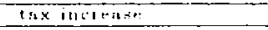 & 36.223 & 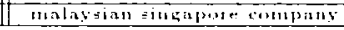 & 30.20 .96 \\
\hline \multicolumn{6}{|c|}{ Topic 10 Siginature Terms of Topic 258 Computer Security } \\
\hline Tnignam & $-2 \log \lambda$ & Mikram! & $-2 I_{13 g \lambda}$ & Thigram & $-2 \log x$ \\
\hline computer & 11513.351 & componter secousty & 213.331 & jet propulasion laberatioy & 14.854 \\
\hline Virus: & 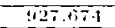 & Fratluate stubtent & $156.5 \mathrm{Hr}$ & Ioberit t min & $\operatorname{ses} \times 54$ \\
\hline Tiackint & $8 \times 7.375$ & computer $3 y=10 \mathrm{~m}$ & $1+16.32 \mathrm{~K}$ & cormell university sraduate & 79081 \\
\hline inerivia & tifiti 392 & Iesmaich couter & 132.113 & 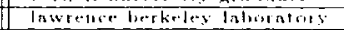 & 79.01 \\
\hline Eirmeli & $3 \times 5 \cdot \operatorname{tix} \cdot 1$ & Gompuler virus & 126.0333 & What jet propulasion & $70.0 \times 1$ \\
\hline Muyessity & 305.958 & corbejl tubersity & $108 .+11$ & Hadversty graduate etudeni & -79051 \\
\hline system & 290.347 & uncleat weraput & $107.2 \mathrm{B3}$ & Thwreuce liveromoge fiational & 60105 \\
\hline Taborataty & 287.521 & military componter & $10 t .52 \div 2$ & Tivernoure ntotiotal laboratory & $10190 \mathrm{t}$ \\
\hline $\mathrm{Tab}$ & 205.511 & visus prograsle & $106, .522$ & computer seculty expert & 86.190 \\
\hline meselary & $12 \times .515 \mathrm{~s}$ & weit getman & 82210 & security center bethesda & 49.123 \\
\hline \multicolumn{6}{|c|}{ Topic 10 Signature Terins of Topic $271-$ Solar Power } \\
\hline Tinikrain & $-3 \log \lambda$ & Biptian & $-2 \log \lambda$ & Trigrnim & $-3 / 0,1 \mathrm{~A}$ \\
\hline solat & $1 \times 1.315$ & Solar enetgy & $2 \operatorname{2in} .521$ & divisinu multiple acess & 31.347 \\
\hline Tmzilin & 301015 & scolat prower & 91.210 & mobile: telephathe serviere & 31.317 \\
\hline Ten & $2 \pi+1332$ & rhristian ind & कीi.211 & butial terhenology group & 2351010 \\
\hline iriclinin... & $25 \mathrm{sh}, 6105$ & teis $x y+t+0$ & 611.535 & 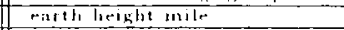 & 23.510 \\
\hline pravilimen & $0101 \mathrm{MI1}$ & moluble telephesine & in 535 & fincincial hackins iridum & 23.510 \\
\hline montind & $12 n, 121$ & iridiun project & 6.6197 & Glotiol mothile satedlite & 23510 \\
\hline tower & 196.35 & tat Encil: & 61.0161 & 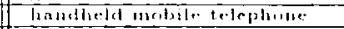 & 93510 \\
\hline Trickuinit & $22 \pi+10 t i$ & scientan patk & 31. nos & Mobile satallate ystem & 23.510 \\
\hline I11marsat & $109.72 \mathrm{X}$ & oncentratoit & $51 . M 59$ & motorola iridium penject & 23.510 \\
\hline hoovdstont & $2 \times .373$ & 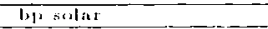 & 31.3 .77 & netivestaln aystem & 15.673 \\
\hline
\end{tabular}

Table 2: Top 10 signature terms of unigram, bigram, and trigram for four TREC topies.

\subsection{Comparing Summary Extraction Effectiveness Using Topic Signatures, TFIDF, and Baseline Algorithms}

In order to evaluate the effectiveness of topic signaltures used in summary extraction, we compare the summary sentences extracted by the topic signature module, bascline module, and ifidf modules with hinmain annotated model summaries. We measure the performance using a combined measure of recall $(R)$ and precision $(P), F$. F-score is defined by:

$$
F=\frac{\left(1+\beta^{2}\right) P^{\prime} R}{\beta^{2} P^{P}+R}, \text { where }
$$

$$
\begin{aligned}
& R=\frac{N_{m e}}{N_{m}} \\
& P=\frac{N_{m e}}{N_{r}} \\
& N_{m} \text { : \# of sentences extrated that also } \\
& \text { apperar in the model summary } \\
& N_{m} \text { : \#t of sentences in the model summary } \\
& N_{:}: \text {: \# of sentences extructed by the system } \\
& \beta \text { : relative importance of } R \text { and } P
\end{aligned}
$$


markups. The munber of docunnents with answer keys are listed in the row labeled: "\# of Relevant Doss Used in Training". To ensure we utilize all the available data and conduct a somnd evaluation, we perform a three-fold cross valiclation. We roserve one-third of documents as test set, use the rest as training set, and repeat three times with nonoverlapping test set. Furthernore, we use only unigram topic signatures for evaluation.

The result is shown in Figure 2 and Table 3 . We find that the topic signature method ontperforms the other two methods and the tfidf method performs poorly. Among 40 possible test points for four topics with $10 \%$ summary length increment (0\% means select at least one sentence) as shown in Table 3 , the topic signature metlod beats the baseline method 34 times. This result is really encouraging and indicates that the topic signature method is a worthy addition to a variety of text summarization methods.

\subsection{Enriching Topic Signatures Using Existing Ontologies}

We have shown in the previous sections that topic signatures can be used to approximate topic identification at the lexical level. Although the automatically acquired signature terms for a specific topic seem to be bound by unknown relationships as shown in Table 2, it is hard to image how we can enrich the inherent flat structure of topic signatures as defined in Equation 1 to a construct as complex as a MUC template or script.

As discussed in (Agirre et al., 2000), we propose using an existing ontology such as SFNSUS (Knight and Luk, 1994) to identify signature (erm relations. The external hicrarchical framework can be used to generalize topic signatures and suggest, richer representations for topic signatures. Automated entity rerognizers can be used to classify unknown entities into their appropriate SENSUS concept nodes. We are also investigating other approaches to automatically learn signature term relations. The idea mentioned in this paper is just a starting point.

\section{Conclusion}

In this paper we presented a procedure to automatically acquire topic signatures and valuated the effectiveness of applying topic signatures to extract topic: relevant sentences against two other nethods. The topic signature method ontperforms the baseline and the tfidf methods for all test ropics. Tonic signatures can not only recognize related terms (topic identifi(ation), but group related terms together under one target concept (topic interpretation). Topic: identification and interpretation are two essential steps in a typical automated text summarization system as we present in Section 3.

Topic: signatmes can also been viewed as an inverse process of query expansion. Query axpansion intends to alleriate the word mismatch problem in information retrieval, since documents are normally written in different vocabulary. How to automatically identify highly correlated terms and use them to improve information retrieval performance has been a main research issue since late 1960's. Recent advances in the query expansion (Xu and Croft., 1996) wan also shed some light on the croation of topic signatures. Although we focus the use of topic: signatures to aid text summarization in this paper, we plan to explore the possibility of applying topic signatures to perform query expansion in the future.

The results reported are encouraging enough to allow us to continue with topic signatures as the vehicle for a first approximation to word knowledge. We are now busy creating a large number of signatures to overcome the world knowledge acquisition problem and use them in topic: interpretation.

\section{Acknowledgements}

We thank the anonymous reviewers for very useful suggestions. This work is supported in part by DARPA contract N66001 97-9538.

\section{References}

Eneko Agirre, Olatz Ansa, Eduard IIory, and Davirl Martinez. 2000. Enriching very large ontologies using the www. In Procecdings of the Workshop on Ontology Construction of the European Conference of $A I$ ( $E C A I)$.

Kemneth Church and Patrick Hanks. 1990. Word association norms, mutual information and lexicography. In Proceedings of the 28th Annual Mecting of the Association for Computational Linguistics (ACL-90): pages 76-83.

Thomas Cover and Joy A. Thomas. 1991. Elements of Information Theory. John Wiley \& Sons.

Gerald DeJong. 1982. An overview of the FlRUMIP system. In Wendy G. Lehnert and Martin II. Ringle, editors, Strategies for natural languagc processing, pages 149-76. Lawrence Erlbaum A.ssociates.

led Dumning. 1993. Accurate methods for the statistics of surprise and coincidence. Compulational Linguistics, 19:61-71.

Marti Hearst. 1997. Text'Tiling: Segmentiner text into multi-paragraph subtopic passages. Computational Linguistics, 23:33-64.

Filuard Hovy and Chin-Yow Lin. 1999. Automated text summarization in SUMMARIST. In Inderjeet Mani and Mark T. Maybury, editors, Advances in Automatic Text Summarization, chapter 8, pages 81-94. MII' Puess.

Kevin Knight and Steve K. Iuk. 1994. Building a large knowledge base for machine translation. In Proccedings of the Eleventh Nationul Conference. on Artificial Intelligence (AAAI.94). 


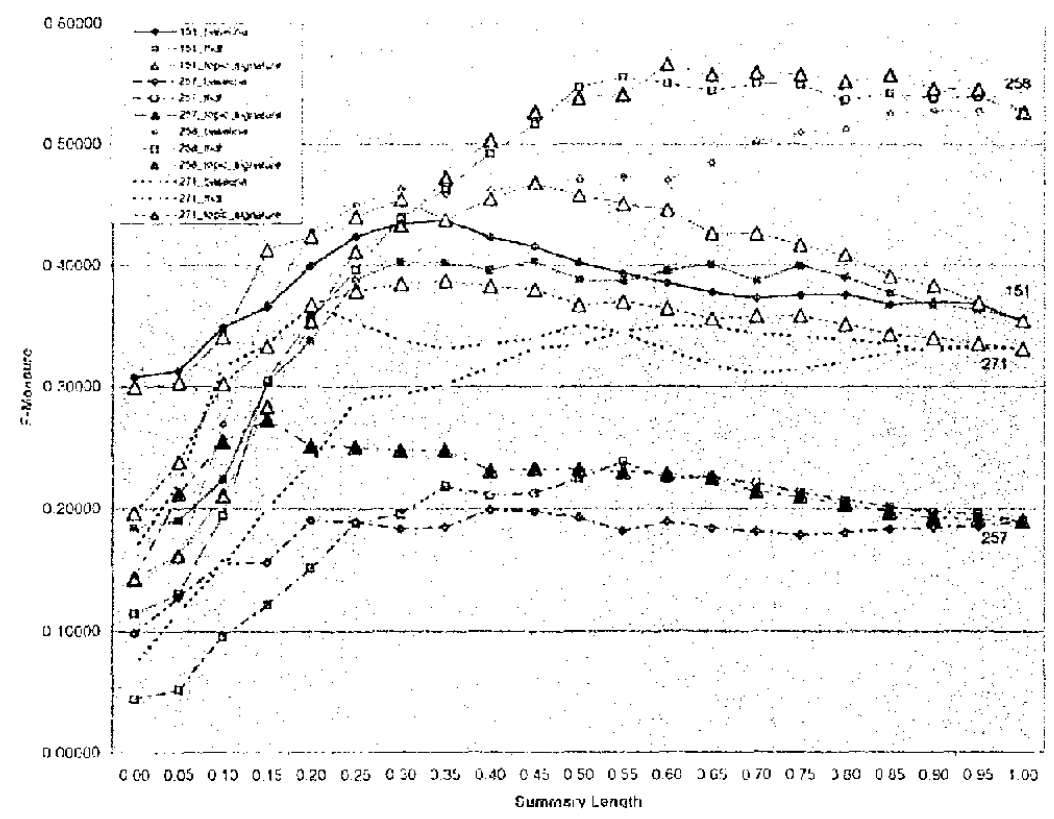

Figure 2: F-measure simmary length for all four topics. Topie signature clearly outperform tfidf and baseline exept for the case of topic 258 where performance for the three nethods are roughly equal.

\begin{tabular}{|c|c|c|c|c|c|c|c|c|c|c|c|}
\hline & $0 \%$ & $10 \%$ & $20 \%$ & $30 \%$ & $10 \%$ & $50 \%$ & $00 \%$ & $70 \%$ & $80 \%$ & $80 \%$ & $100 \%$ \\
\hline 151 -basedinio & 0.3105 & (3.3-19 & 5.400 & $01+134$ & i). 123 & 10.403 & $10.3 \times 5$ & 0.373 & 0378 & (1).371 & C. 355 \\
\hline Lol-tfitif & -30.93 & 35.42 & 15,26 & $-7.2 ! 2$ & -8.35 & 351 & 2.5 .5 & $3 . \overline{7} i$ & $3 \times 3$ & -0.75 & 0.00 \\
\hline $151-1$ & -27 & .0 .19 & +6.02 & +4.58 & $+7.4 \mathrm{~B}$ & +13.77 & $+15 . \overline{83}$ & 14.17 & +8.66 & +3.50 & 0.00 \\
\hline 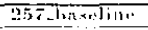 & antis & $\pi 155$ & 0.131 & $\pi .1 \times 1$ & 6.190 & 0.193 & $0.18 \div$ & 0.181 & $0.1 \overline{81}$ & 0.185 & 0.100 \\
\hline $95 \bar{t}$-tfint & -5511 & -336.56 & $20.5 ! 1$ & -16.54 & A. Hon & $41+1.4-1$ & $7 \times .3 .1$ & (1) & +1410 & +700 & 0.00 \\
\hline 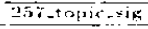 & +45.53 & +84.08 & +31.88 & +34.91 & +16.07 & +20.40 & +20.60 & +18.01 & +12.48 & $+4.2 \pi$ & 0.001 \\
\hline 258 -bitsmlines & $0.1 .1 !$ & 0.270 & 0.128 & $a+3153$ & ii +60 & $01 .+71$ & 6. 1.70 & 11602 & 11.512 & $072 \mathrm{~B}$ & $0.5 \div$ \\
\hline $\operatorname{lin} x$ & $-1 \kappa k-1$ & $-27 \mathrm{ks}$ & -16.57 & 5.21 & iti.tii & $+10 . \overline{18}$ & +16.90 & ini & +1.7 .4 & +1.92 & $0 \%$ \\
\hline 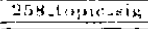 & +1.55 & $=21.82$ & -17.19 & -1656 & +9.96 & +14.18 & +20.40 & +11.44 & +7.74 & +3.43 & c..00 \\
\hline 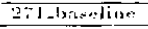 & $0.16 \%$ & 0.310 & it.istix & 1].3.35 & 6136 & 61.351 & 0.331 & 0.410 & 0.321 & 0.333 & 0.332 \\
\hline $2 \pi 1+$ fint & $-5+i \quad 75$ & .31 .35 & -35.5 & 13.2 .2 & $-5,14$ & 1.83 & $T 396$ & +119.97 & 15.7 & -1.2 & i) 010 \\
\hline $2=1+1+1010$ & $+17 . \overline{9 \%}$ & $-\overline{4} \cdot 40$ & to.0e & +13.67 & +14.10 & +4.83 & +10.28 & +15.70 & +9.05 & +2.29 & i. 00 \\
\hline
\end{tabular}

Table 3: F-measure performance difference compared to baseline method in percentage. Columns indicate at different summary lengthis rolatexl to full length documents. Values in the baseline rows are F-measure scores. Values in the lfidf and topie signatners rows are perfomance increase on decrease divided by their corresponding baseline scores and shown in percentage.

Inderject Mani, David House, Gary Klein, Jynotte Mirschman, Leo Obrst, 'Théresese Fimin, Michae? Chrzanowski, and Beth Sumbein. 1998. The TIPSTER SUMMAC text summatization caluation final report. Technical Report MTR98W0000138, The MITRE Corporation.

Christopher Manning and Hinrich Schïtze. 1999. Foudations of Statistical Natural Language Pro. cessing. MI'T Press.

Kathleen McKeown and Dragomir R.. Raddev. 1999. Generating stmmeries of multiple news anticless. In Inderjeet Mani and Mark 'T. Maybury, exlitors, Advances in Automatix That Summerizution, (latapter 24, pages 381 389. MlT Press.

Ellon Riloff and Jelfey Lorenzen. 1999. Extraction- based text ategorization: Generating domain. specifir: roln relationships automatically. In Tomek Strzalkowski, editor, Natural Language Information lietritewal. Khwer Acadenic Publishers.

Elen Riloff. 1996. An empirical study of automated dictionary construction for information extraction in three domains. Artificial Intelligenes. Journal, 85, August.

SAIC. 1998. Introduction to information extraction. http://www muc..saics.com.

Jinxi Xu and W. Bruce Croft. 1996. Query expansion using local and globsal document analysis. In Procecdings of the 17th Anmual International ACM SIGIR Conference on Research and Development in Information lietrieval, pages 411 . 